\title{
ESTUDO DA PREVALÊNCIA DE LESÕES CENTRAIS DOS MAXILARES DIAGNOSTICADAS NO LABORATÓRIO DE PATOLOGIA BUCAL DA UNIVERSIDADE ESTADUAL DE FEIRA DE SANTANA, NO PERÍODO DE 2006 A 2017
}

\section{Rafael Cabral de Albuquerque Souza ${ }^{1}$; Jener Gonçalves de Farias ${ }^{2}$; Tarsila de Carvalho Freitas Ramos ${ }^{3}$; Márcio Campos Oliveira ${ }^{4}$; Valéria Souza Freitas ${ }^{5}$}

1.Bolsista PROBIC/UEFS, Graduando em odontologia, Universidade Estadual de Feira de Santana, email: rafael cabral27@live.com

2.Orientador, Departamento de Saúde, Universidade Estadual de Feira de Santana, email: jenerfarias@gmail.com

3.Pesquisadora do Núcleo de Câncer Oral, Departamento de Saúde, Universidade Estadual de Feira de Santana, email: professoratarsila@yahoo.com.br

4. Pesquisador do Núcleo de Câncer Oral, Universidade Estadual de Feira de Santana, email: marciopatologiaoral@gmail.com

5. Pesquisa e Coordenadora do Núcleo de Câncer Oral, Universidade Estadual de Feira de Santana, email: valeria.souza.freitas@gmail.com

\section{Introdução}

Palavras-chave: Epidemiologia; Medicina Bucal; Estomatologia

De forma resumida, as patologias que podem ser encontradas no sistema estomatognático são divididas em dois grandes grupos: as lesões periféricas e as lesões centrais (NEVILLE et al., 2009). As lesões periféricas são entendidas por lesões que se encontram fora das corticais ósseas (MENDEZ et al., 2012). Já as lesões centrais se encontram dentro das corticais ósseas e incluem os cistos odontogênicos e não odontogênicos, os tumores odontogênicos e não odontogênicos e as lesões associadas ao osso (NEVILLE et al., 2009). As lesões centrais dos maxilares possuem em seu diagnóstico um elevado grau de dificuldade, uma vez que a maioria costuma ser assintomática e de crescimento lento e, em muitas situações, é observada por meio de exames radiográficos de rotina, a exemplo da radiografia panorâmica solicitada em uma documentação para planejamento ortodôntico (DE CARVALHO; PEDREIRA, 2016). Dentre os grupos de lesões centrais com maior frequência de acometimento nos maxilares, podem ser citados: 1 - cistos odontogênicos e não-odontogênicos, como o cisto radicular, dentígero e nasopalatino; 2 - tumores odontogênicos e não odontogênicos, como o odontoma, ameloblastoma e osteoma e 3 - as lesões associadas ao osso - como as displasias fibro-ósseas, cemento-ósseas e o fibroma ossificante central (PEREIRA et al., 2010). A quantidade de estudos voltados à epidemiologia das lesões bucais é baixa quando comparada aos que se direcionam a cárie e a doença periodontal, ainda que a Organização Mundial de Saúde os preconize. As diretrizes brasileiras da Política Nacional de Saúde Bucal não têm inserido no seu programa o rastreamento de alterações de normalidade e de lesões do sistema estomatognático, tão importante para o desenvolvimento de um perfil epidemiológico do país (DE MELO et al., 2013).

\section{Metodologia}

Foi realizado um estudo de corte transversal onde se verificou todos os prontuários dos pacientes com diagnóstico de lesão intraóssea entre os anos de 2006 e 2017, comprovados através de resultado do exame histopatológico, sendo esses os critérios de inclusão da amostra, no Centro de Referência de Lesões Bucais (CRLB) da Universidade Estadual de Feira de Santana/Bahia. Os dados foram coletados através de uma planilha de coleta contendo informações sobre sexo; idade; diagnóstico histopatológico; localização anatômica. As informações foram sistematizadas em 
tabelas e gráficos com auxílio do Programa Excel da MICROSOFT CORPORATION (2003), seguindo a seqüência e distribuição das variáveis do estudo. Os dados foram digitados e analisados utilizando o programa Statistical Package for Social Science SPSS, versão 10.0, sendo apresentadas distribuições absolutas e percentuais. Para a análise bivariada, a normalidade dos dados contínuos foi analisada com o teste de Kolmogorov-Smirnov. Em seguida, foram empregados os testes não-paramêtricos Quiquadrado de Pearson (para variáveis categóricas) e Kruskall-wallis seguido de Mannwthiney com correção de Bonrrefoni (para variáveis contínuas). Foram consideradas diferenças estatisticamente significantes as diferenças com valor de $\mathrm{p} \leq 0.05$.

\section{Resultados e discussão}

Durante o período de estudo, o CRLB realizou 2051 biópsias de lesões bucais, sendo que as lesões intraósseas representaram $14,1 \%(\mathrm{n}=290)$ da população. O grupo dos cistos $(47,58 \%, n=138)$ obteve o maior número de diagnósticos realizados pelo centro. Quanto as características sociodemográficas, a maioria dos indivíduos foram do sexo feminino $(68,7 \%, n=198)$ e encontravam em sua maioria na $2^{\mathrm{a}}$ e $4^{\mathrm{a}}$ décadas de vida. Já para a variável clínica localização anatômica, a maior parte das lesões foram diagnosticadas na região posterior de mandíbula $(42,4 \%, n=123)$. No grupo dos cistos observa-se uma idade média de 40 anos de idade, sendo que a $4^{\text {a }}$ década de vida é a faixa etária mais prevalente. A frequência do sexo feminino foi maior entre os indivíduos com o diagnóstico de cisto, quando comparado com os demais $(p=0,005)$. $O$ sítio anatômico onde obteve o maior número de caso para este grupo foi a região posterior de mandíbula. O sexo feminino foi o mais acometido pelas lesões intraósseas, o que corrobora com o estudo de Phattaratarip et al. realizado na Universidade Chulalongkorn em Bangkok, Tailândia. Porém, alguns outros estudos evidenciaram o sexo masculino como o mais prevalente (KEBEDE et al., 2017; LAWAL; SOYELE; AKINYAMOJU, 2015; RAMACHANDRA et al., 2014; VILLASIS-SARMIENTO; PORTILLA-ROBERTSON; MELENDEZ-OCAMPO, 2017). Essas divergências podem ser explicadas, pelo fato dos estudos envolverem grupos de lesões específicos com suas determinadas características e este trabalho abrange todas as lesões intraósseas. A faixa etária com o maior número de casos foi entre a $2^{\mathrm{a}}$ e $4^{\mathrm{a}}$ década de vida, portanto estes achados estão de acordo com encontrados na África (MUWAZI; KAMULEGEYA, 2015), Oriente Médio (ALSHEDDI; ALSENANI; ALDOSARI, 2015), Ásia (KAMBALIMATH et al., 2014; NALABOLU et al., 2017) e América e Europa (JOHNSON et al., 2013; LOUREDO et al., 2017).

Gráfico 1. Distribuição do número (n) de casos das cinco lesões intraósseas mais frequentes, segundo o diagnóstico histopatológico, CRLB/UEFS, Feira de Santana-BA, Brasil, 2006-2017 


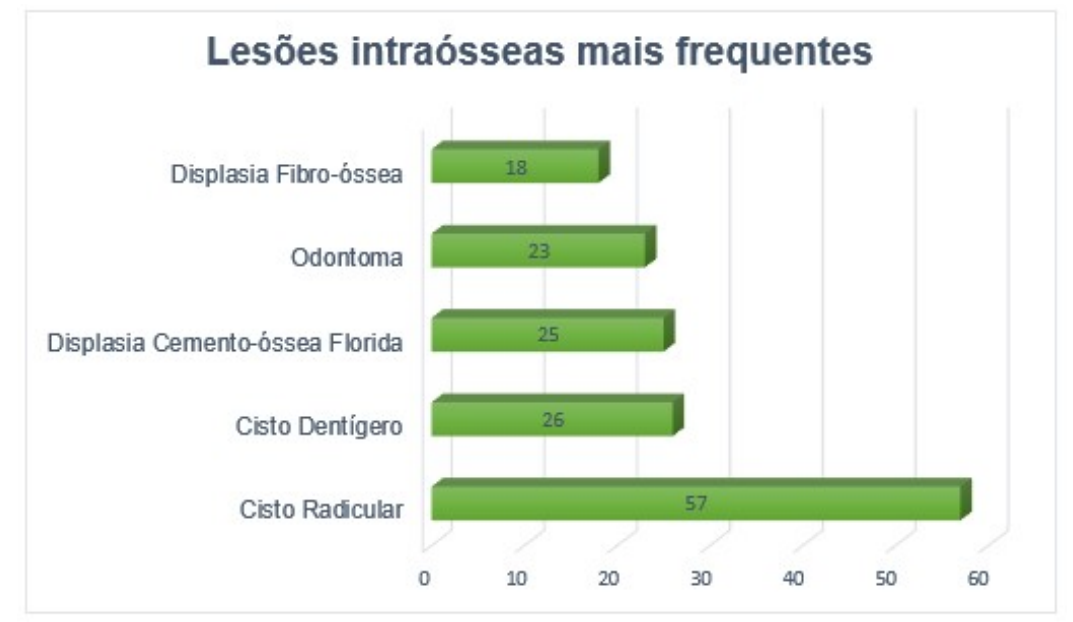

Tabela 1. Análise bivariada para os cistos com as variáveis sexo, localização anatômica e faixa etária, CRLB/UEFS, Feira de Santana-BA, Brasil, 2006-2017

\begin{tabular}{|c|c|c|c|c|c|}
\hline \multirow[t]{2}{*}{ Variáveis } & \multicolumn{2}{|c|}{ Cistos } & \multicolumn{2}{|c|}{$\begin{array}{l}\text { Lesões não } \\
\text { císticas }\end{array}$} & \multirow[t]{2}{*}{ Valor de $p$} \\
\hline & $\mathbf{N}$ & $\%$ & $\mathbf{N}$ & $\%$ & \\
\hline \multicolumn{6}{|l|}{ Sexo } \\
\hline Masculino & 55 & 39,9 & 37 & 24,3 & \multirow{2}{*}{$0,005^{\star}$} \\
\hline Feminino & 83 & 60,1 & 115 & 75,7 & \\
\hline \multirow{2}{*}{\multicolumn{6}{|c|}{$\begin{array}{l}\text { Localização } \\
\text { anatômica }\end{array}$}} \\
\hline & & & & & \\
\hline Mandíbula Anterior & 20 & 14,5 & 24 & 15,8 & \multirow{4}{*}{0,071} \\
\hline Mandíbula Posterior & 55 & 39,9 & 68 & 44,7 & \\
\hline Maxila Anterior & 44 & 31,9 & 29 & 19,1 & \\
\hline Maxila Posterior & 19 & 13,8 & 31 & 20,4 & \\
\hline \multicolumn{6}{|l|}{ Faixa Etária ${ }^{1}$} \\
\hline $0-10$ & 6 & 4,4 & 8 & 5,3 & \\
\hline $11-20$ & 17 & 12,4 & 31 & 20,5 & \\
\hline $21-30$ & 24 & 17,5 & 21 & 13,9 & \\
\hline $31-40$ & 27 & 19,7 & 33 & 21,9 & 0,15 \\
\hline $41-50$ & 21 & 15,3 & 12 & 7,9 & \\
\hline $51-60$ & 19 & 13,9 & 18 & 11,9 & \\
\hline $61-70$ & 14 & 10,2 & 16 & 10,6 & \\
\hline $71-80$ & 6 & 4,4 & 12 & 7,9 & \\
\hline $81-90$ & 3 & 3,3 & 0 & 0,0 & \\
\hline
\end{tabular}

(1) 2 Fichas sem informação, * valor de $\mathrm{p}=0,05$

Tabela 2. Distribuição dos indivíduos acometidos por lesões intraósseas e seus grupos de acordo com sexo, idade e localização anatômica, CRLB/UEFS, Feira de Santana-BA, Brasil, 2006-2017 


\begin{tabular}{|c|c|c|c|c|c|c|c|c|c|c|}
\hline \multirow[t]{2}{*}{ Variáveis } & \multicolumn{2}{|c|}{$\begin{array}{c}\text { Lesões } \\
\text { intraósseas }\end{array}$} & \multicolumn{2}{|c|}{ Cistos } & \multicolumn{2}{|c|}{$\begin{array}{c}\text { Tumores } \\
\text { odontogênicos }\end{array}$} & \multicolumn{2}{|c|}{$\begin{array}{l}\text { Lesões } \\
\text { associadas } \\
\text { ao osso }\end{array}$} & \multicolumn{2}{|c|}{$\begin{array}{c}\text { Doenças do } \\
\text { periápice e } \\
\text { outros }\end{array}$} \\
\hline & N & $\%$ & $\mathbf{N}$ & $\%$ & $\mathbf{N}$ & $\%$ & $\mathrm{~N}$ & $\%$ & $\mathrm{~N}$ & $\%$ \\
\hline \multicolumn{11}{|l|}{ Sexo } \\
\hline Masculino & 92 & 31,3 & 55 & 39,9 & 15 & 30,6 & 12 & 17,6 & 8 & 30,8 \\
\hline Feminino & 198 & 68,7 & 83 & 60,1 & 34 & 69,4 & 56 & 82,4 & 18 & 69,2 \\
\hline \multicolumn{11}{|l|}{ Idade $^{1}$} \\
\hline 00-10 & 13 & 4,5 & 6 & 4,4 & 6 & 12,5 & 1 & 1,5 & 0 & 0,0 \\
\hline $11-20$ & 48 & 16,6 & 17 & 12,4 & 20 & 41,7 & 10 & 14,7 & 1 & 3,8 \\
\hline $21-30$ & 45 & 15,6 & 24 & 17,5 & 6 & 12,5 & 5 & 7,4 & 10 & 38,5 \\
\hline $31-40$ & 60 & 20,8 & 27 & 19,7 & 9 & 18,8 & 10 & 25 & 7 & 26,9 \\
\hline $41-50$ & 32 & 11,1 & 21 & 15,3 & 4 & 8,3 & 5 & 7,4 & 2 & 7,7 \\
\hline $51-60$ & 34 & 11,8 & 19 & 13,9 & 2 & 4,2 & 12 & 17,6 & 1 & 3,8 \\
\hline $61-70$ & 29 & 10 & 14 & 10,2 & 0 & 0,0 & 12 & 17,6 & 3 & 11,5 \\
\hline $71-80$ & 15 & 5,2 & 6 & 4,4 & 1 & 2,1 & 6 & 8,8 & 2 & 7,7 \\
\hline $81-90$ & 3 & 1 & 2 & 2,2 & 0 & 0,0 & 0 & 0,0 & 0 & 0,0 \\
\hline \multicolumn{11}{|c|}{$\begin{array}{l}\text { Localização } \\
\text { Anatômica }\end{array}$} \\
\hline $\begin{array}{l}\text { Mandíbula } \\
\text { Anterior }\end{array}$ & 44 & 15,1 & 20 & 14,5 & 11 & 22,4 & 11 & 16,2 & 1 & 3,8 \\
\hline $\begin{array}{l}\text { Mandíbula } \\
\text { Posterior }\end{array}$ & 123 & 42,4 & 55 & 39,9 & 16 & 32,7 & 37 & 54,4 & 9 & 34,6 \\
\hline $\begin{array}{l}\text { Maxila } \\
\text { Anterior }\end{array}$ & 73 & 25,1 & 44 & 31,9 & 16 & 32,7 & 5 & 7,4 & 8 & 30,8 \\
\hline $\begin{array}{l}\text { Maxila } \\
\text { Posterior }\end{array}$ & 50 & 17,2 & 19 & 13,8 & 6 & 12,2 & 15 & 22,1 & 8 & 30,8 \\
\hline
\end{tabular}

\section{Conclusão}

As lesões estudadas foram mais comuns nas mulheres entre a segunda e quarta década de vida. O grupo de lesões mais frequente foram os cistos odontogênicos com maior predileção para o sexo feminino com idade média de 40 anos e a região posterior de mandíbula como localização principal. Já o diagnóstico histopatológico mais comum foi o cisto radicular, seguida do cisto dentígero, displasia cemento-óssea florida, odontoma e displasia fibro-óssea. O sexo, a idade e a localização anatômica podem ser fatores associadas para alguns grupos de lesões intraósseas como a relação entre o sexo e os cistos.

\section{Referências}

ALSHEDDI, M. A.; ALSENANI, M. A.; ALDOSARI, A. W. Odontogenic tumors: Analysis of 188 cases from Saudi Arabia. Annals of Saudi Medicine, v. 35, n. 2, p. 146-150, 2015.

DE CARVALHO, F. C. R.; PEDREIRA, F. R. DE O. A interferência das lesões ósseas dos maxilares na Ortodontia. Revista Clinica de Ortodontia Dental Press, v. 15, n. 1, p. 81-90, 2016.

DE MELO, A. U. C. et al. Aspectos metodológicos de estudos de prevalência de lesões da mucosa bucal: possibilidade de aplicação em levantamentos nacionais. Clipeodonto, v. 5, n. 1, p. 42-50, 2013.

JOHNSON, N. R. et al. A prospective epidemiological study for odontogenic and non-odontogenic lesions of the maxilla and mandible in Queensland. Oral Surgery, Oral Medicine, Oral Pathology and Oral Radiology, v. 115, n. 4, p. 515-522, 2013.

KAMBALIMATH, D. H. et al. Prevalence and Distribution of Odontogenic Cyst in Indian Population: A 10 Year Retrospective Study. Journal of Maxillofacial and Oral Surgery, v. 13, n. 1, p. 10-15, 2014.

KEBEDE, B. et al. Odontogenic tumors in Ethiopia: Eight years retrospective study. BMC Oral Health, v. 17, n. 1, p. 1-7, 2017.

LAWAL, A. O.; SOYELE, O. O.; AKINYAMOJU, A. O. A retrospective study of 21 cases of malignant odontogenic tumours from two tertiary health centres in Nigeria. Pan African Medical Journal, v. 20, p. $1-5,2015$.

LOUREDO, B. V. R. et al. Estudo epidemiológico de lesões odontogênicas provenientes do Departamento de Patologia e Medicina Legal da Universidade Federal do Amazonas. Revista Brasileira Odontologica, v. 74, n. 2, p. 126-132, 2017.

MENDEZ, M. et al. A 10-year study of specimens submitted to oral pathology laboratory analysis: lesion occurrence and demographic features. Brazilian Oral Research, v. 26, n. 3, p. 235-241, 2012. 
MUWAZI, L. M.; KAMULEGEYA, A. The 5-year prevalence of maxillofacial fibro-osseous lesions in Uganda. Oral Diseases, v. 21, n. 1, p. 79-85, 2015.

NALABOLU, G. R. K. et al. Epidemiological study of odontogenic tumours: An institutional experience. Journal of Infection and Public Health, v. 10, n. 3, p. 324-330, 2017.

NEVILle, B. W. et al. Patologia Oral e Maxilofacial. 3. ed. Rio de Janeiro: Elsiever, 2009.

PEREIRA, J. V. et al. Prevalência de cistos e tumores odontogênicos em pacientes atendidos na Fundação Assistencial da Paraíba: estudo retrospectivo. Arquivos em Odontologia, v. 46, n. 2, p. 75-81, 2010.

RAMACHANDRA, S. et al. Prevalence of odontogenic cysts and tumors: A retrospective clinicopathological study of 204 cases. SRM Journal of Research in Dental Sciences, v. 5, n. 3, p. 3-6, 2014. VILLASIS-SARMIENTO, L.; PORTILLA-ROBERTSON, J.; MELENDEZ-OCAMPO, A. Prevalence and distribution of odontogenic cysts in a Mexican sample . A 753 cases study. Journal Clinical Exp Dent, v. 9, n. 4, p. 531-538, 2017. 